\title{
Revista Chilena de Pediatria: Desde el papel al mundo digital
}

\author{
Revista Chilena de Pediatria: from the paper to digital world
}

\author{
Francisco Cano Sch. ${ }^{\mathrm{a}}$, Paul Harris D. ${ }^{\mathrm{a}}$, Luisa Schonhaut B. ${ }^{\mathrm{a}}$
}

${ }^{a}$ Comité Editorial Revista Chilena de Pediatría

\section{Introducción}

Hace décadas, un grupo de visionarios pediatras reunidos en la Sociedad Chilena de Pediatría definieron un camino que hoy parece de absoluta obviedad, no así en esa época, en que la medicina y la sociedad eran completamente distintas al día de hoy. Ellos definieron, hace casi 90 años, que la experiencia y el conocimiento científico debía ser trasmitido por escrito, de generación en generación, para que todo médico pudiese conocer y aplicar esa enseñanza al momento del diagnóstico y tratamiento de sus pacientes.

Así nació Revista Chilena de Pediatría. En su primer número el año 1930, bajo la dirección del Dr. Eugenio Cienfuegos, se publicaban artículos como Distrepsias de origen psíquico, vacunación antidiftérica y Glomerulonefritis isquémica difusa, entre muchos otros ${ }^{1}$.

Esta visionaria iniciativa puede hoy ser comprendida a la luz de una pregunta...

¿Por qué la investigación y la publicación científica son los pilares del avance del conocimiento en medicina $y$ en la sociedad?

Durante largos periodos de tiempo, la economía y el desarrollo de los países se basaba principalmente en recursos agrícolas. Mas tarde, la era agrícola fue reemplazada por la era industrial, y la economía global y el desarrollo pasaron a depender de la generación de recursos manufactureros como el carbón, acero, petróleo, todas fuentes de energía para la producción. Hoy sabemos que ese tipo de recursos, en tanto más de usan, se agotan. No son renovables, y en una época en que la palabra crecimiento define las metas de nuestra sociedad, multiplicando permanentemente los objetivos una vez alcanzadas las metas, sin duda los recursos materiales tienen una fecha de expiración.

En contraste, el recurso que visionariamente nos legaron pediatras como Cienfuegos, Calvo Mackenna, Roberto del Río, entre tantos otros, el Conocimiento, no se agota. Por el contrario, en tanto más lo usamos y desarrollamos, más se genera. "El mayor de los descubrimientos científicos ha sido el de la ignorancia. Cuando los humanos se dieron cuenta de lo poco que sabían acerca del mundo, tuvieron una muy buena razón para buscar nuevo conocimiento, lo cual abrió el camino científico hacia el progreso" (Harari Y.N. Homo Deus: Breve historia del mañana. 2015).

De esta forma, el conocimiento y su principal camino de globalización, la publicación científica, es un recurso que se autoperpetúa, retroalimenta y multiplica. El futuro de las sociedades humanas no está en los recursos agotables, está en la generación de conocimiento. El conocimiento ha sido la base de los avances en medicamentos, en tecnología, en biología, en terapia génica, en medicina traslacional, en Inteligencia Artificial, todo lo cual ha abierto caminos cuyo alcance no logramos ni siquiera a imaginar. En tanto mayor

Correspondencia:

Luisa Schonhaut

Ischonhaut@yahoo.com 
conocimiento se alcanza en estos diferentes campos, más se amplía la base para nuevos descubrimientos, y nuevamente para mayor conocimiento.

En este camino la investigación y la publicación científica en Chile han mostrado un crecimiento y fortaleza que poco coincide con la permanente escasez de recursos asignados a nivel central. Con un presupuesto Fondecyt prácticamente congelado por un proyecto de ley de presupuesto 2019 que incrementa en un 0,2\% real el monto asignado a este Fondo de investigación, el importante crecimiento del activo período 20092013 no parece destinado a repetirse (El Mercurio, octubre 15, 2018).

A pesar de ello, a la luz del último informe de la Red Iberoamericana de Indicadores de Ciencia y Tecnología (http://dev.ricyt.org/ui/v3/comparative.html? indicator=CSCI), RICYT, las cifras de publicación científica favorecen ampliamente a nuestro país.

De acuerdo a ese informe, las publicaciones SCI (Science Citation Index, Thomson Reuters, actual Clarivate Analitycs) durante el año 2016 en nuestra Región corresponden para Argentina a una cifra de 11205, Brasil 53819, Chile 10495, Colombia 5692, Uruguay 1246, entre otros. Al corregir estos números por población de países, año 2017, del Banco Mundial (https://datos. bancomundial.org/indicator/SP.POP.1564.TO.ZS), para el rango etario de 15-65 años, aproximadamente $65 \%$ de la población total, en Argentina 43.823.000, Brasil 207.012.000, y Chile 18.280.000 hábs, la publicación científica nacional supera ampliamente a los países de la Región.

$\mathrm{Al}$ ajustar las cifras de publicaciones SCI (RICYT 2017) por el número de artículos/100.000 habitantes, se informa una cifra de 25,7, 26,1 y 57,6 para Argentina, Brasil y Chile respectivamente, donde la publicación científica nacional supera ampliamente a los países de la Región. El análisis de publicaciones SCI ajustados al PBI de cada país muestra una cifra de 20, 30 y 41 para estos 3 países en el mismo orden anterior, en tanto que el número de publicaciones SCI ajustado por gasto I+D para Argentina y Chile informa una cifra de 3,79 y 11,58 respectivamente, sin valores informados para Brasil. Finalmente, las publicaciones en Medline informadas por RICYT para el año 2017 muestran un número de 5167 para Argentina, 29181 para Brasil, y 3901 para Chile, cifras que al aplicar una corrección sobre población mantienen una diferencia nacional positiva comparada con la Región.

Este camino sin embargo, no está exento de peligros, y debe ser mirado tanto desde el punto de vista científico, como humanista. Como tantas cosas, en una sociedad de mercado, en una "sociedad del cansancio" (Die Mudigkeitsgesellschaft, Han B.Ch. 2010) en que una sociedad disciplinaria ha sido reemplazada por una sociedad de rendimiento, donde el "exceso de trabajo y de rendimiento se agudiza y se convierte en una autoexplotación", las motivaciones han cambiado. Peor aún si la racionalidad económica, la que todo lo invade, incluido el arte del cuidar personas, impone indicadores a cumplir y plazos a respetar (Micco S. 2017) $)^{2}$.

La publicación científica actualmente se ha hecho parte de los requerimientos que impone la obtención de grados académicos, de contratos institucionales, de concursos para financiar proyectos de investigación, de la industria en productos relacionados a la salud, o simplemente de empresas cuyo objetivo principal es el lucro.

En este escenario, sabemos que la mayoría de los profesionales que investigan y luego publican sus conocimientos, están motivados por el hecho de trasmitir su conocimiento a los jóvenes médicos en el manejo de los pacientes. Para aquellos que permanentemente entregan lo mejor de su trabajo a través de la publicación científica, analizaremos en las secciones siguientes el escenario editorial global, y el estado actual de Revista Chilena de Pediatría en ese contexto.

\section{Estado actual de las principales bases de datos internacionales de publicación científica}

El mundo de los índices de impacto ha sufrido cambios importantes en la última década. Hemos pasado de un monopolio ejercido por el Factor de Impacto (FI) de Web of Science (WOS) a una amplia gama de índices o scores de evaluación de revistas científicas. Ello se ha debido, entre otras razones, a la necesidad de evaluar las revistas utilizando otras variables, además del convencional índice FI, que integren otros elementos y que amplíen la posibilidad de evaluar las revistas rompiendo el monopolio de WOS.

El FI originalmente pertenecía al Instituto de Información Científica (Institute for Scientific Information, ISI) manejado por la empresa Thompson Reuters, que generó una plataforma integrada (Thomson Reuters Web of Knowledge) que incluye diferentes productos, destacando Conference Proceedings Citation Index, Current Contents, Journal Citation Reports, Medline, Scholar-One Products, Science Citation Index, Science Citation Index Expanded, y Social Sciences Citation Index, entre otros. Esta compleja RED es llamada Web of Science (WOS). Actualmente, WOS ha pasado a manos de un consorcio Canadiense-Chino, Clarivate Analytics, que pretende continuar con la denominación FI.

En términos simples, WOS es la Colección Principal (core collection) y equivalente al antiguo nombre de ISI web of science, donde JCR (Journal Citacion Report) proporciona la información para el cálculo de la 
métrica FI. Así WOS es una base referencial o índice multidisciplinario, que tiene 12.000 revistas más libros y resúmenes, pero no tiene artículos completos, ya que no es no es una biblioteca o un repositorio, sino una plataforma.

El FI es entregado anualmente por el JCR, instrumento que ofrece una manera objetiva y sistemática para evaluar revistas científicas basada en información estadística cuantificable que proviene de datos de citación de los 2 últimos años. Adicionalmente entrega un Índice de Inmediatez (último año), y un Factor de Impacto de 5 años. Se utiliza el " 2 años" porque en ese momento se alcanza el máximo de citas en ciencias médicas, no así en humanidades, y donde el numerador son citas a ítems publicados, es decir, citas recibidas, cualquier material (revisiones, cartas, etc), y el denominador son todos los ítems publicados que tenga resumen y palabras claves.

Afortunadamente, en los últimos año han aparecido nuevos indicadores como: CiteScore, Scimago, Índice, Indice i10, Almetrics, entre otros.

El ranking Scimago permite evaluar a las revistas clasificándolas en cuartiles, y compararlas con revistas de la especialidad, región, etc... y observar los cambios evolutivos en el tiempo. Scimago usa la base de datos llamada Scopus que pertenece a la empresa Elsevier, propiedad de un consorcio Español.

CiteScore, perteneciente a Elsevier, ha surgido como competencia directa de Clarivate, pero usa un rango de 3 años para el cálculo de su factor de impacto y adiciona un elemento dinámico, el CiteScore Tracker, que a diferencia del FI de Clarivate que es estático en el año, permite verificar en forma actual la evaluación métrica de la revista. Adicionalmente incluye en sus evaluaciones todo tipo de manuscritos, con o sin metodología IMRYD.

No podemos dejar de lado a SciELO, base de datos que comprende América Latina, España, Portugal, el Caribe y Sud África, que incluye alrededor de 650 revistas, sobre 4 millones de citas, de acceso abierto y con disponibilidad del texto completo de los manuscritos.

Otros índices también han surgido. Dentro ellos Google, a través de su plataforma Google Scholar, el cual ha incorporado los índices H. Finalmente otras métricas consideran factores de impacto social, es decir su permanencia y presencia en instrumentos de difusión y por tanto analiza las redes sociales. Dentro de ellos, es altmetrics el más difundido.

\section{Indicadores bibliométricos Revista Chilena de Pediatría}

La Revista Chilena de Pediatría fue indexada en año 2014 en Medline, pero aún no cuenta con FI, el cual es entregado por WOS una vez que la revista ha alcanzado al menos 2 años en Medline, no existiendo una ruta oficial de postulación ni procesos conocidos para su permanencia o retiro de dicha plataforma.

En el ranking Scimago, la Revista Chilena de Pediatría ha mostrado un crecimiento continuo, alcanzando el cuartil 3 con índice de 0,171 el año 2017 (figura 1). Mientras que en CiteScore la revista fue catalogada en percentil 28 en el mismo año.

$\mathrm{Al}$ comparar el ranking de las revistas pediátricas latinoamericanas, destaca que la Revista Chilena de Pediatría se encuentra en quinto lugar, siendo superada por 3 revistas brasileñas y por Archivos Argentinos de Pediatría (https://www.scimagojr.com/journalrank.ph $p$ ? category $=2735 \&$ area $=2700 \&$ country $=$ Latin $\% 20 \mathrm{Am}$ erica\&openaccess $=$ true\&scielo $=$ true). En la medida que aumenta la visibilidad de la revista aumenta su flujo y su internacionalización, con un aumento progresivo de los manuscritos recibidos, incrementándose a su vez las tasas de rechazo. Revista Chilena de Pediatría, en la última década, ha incrementado en cerca del 50\% el número de documentos publicados anualmente, mientras que el total de documentos sometidos a revisión se triplicó. Paralelamente se ha recibido y publicado un creciente número de documentos extranjeros (figura 2). Los países que más han participado son Colombia, España y México y en la medida que la revista se está publicando en forma bilingüe, a partir del año 2017, se han recibido manuscritos de países no hispanoparlantes, como Estados Unidos y Alemania, entre otros.

El incremento en el ranking de Revista Chilena de Pediatría ha sido producto de un trabajo constante de un grupo de editores con el apoyo permanente de la Sociedad Chilena de Pediatría, que ha permitido el financiamiento de la revista para mantener un formato de acceso abierto. Además debemos destacar la valiosa contribución de los investigadores, tanto en el rol de

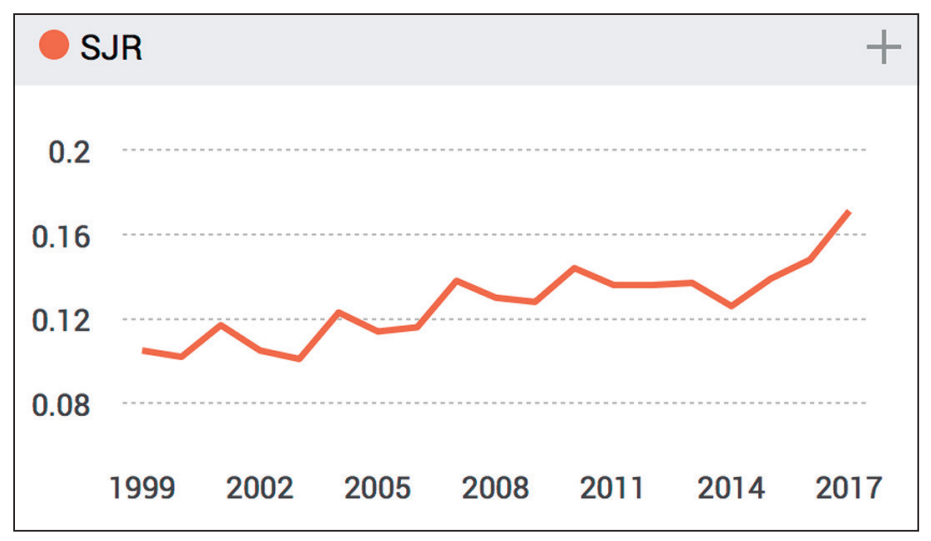

Figura 1. Progresión del Índice SJR de Revista Chilena de Pediatría 1999 a 2017. (https://www.scimagojr.com/journalsearch.php?q=15786 \&tip=sid\&clean=0) 


$$
\begin{aligned}
& 80-80-73-81>_{63-64-61}^{90}>_{72}^{89-95}>
\end{aligned}
$$

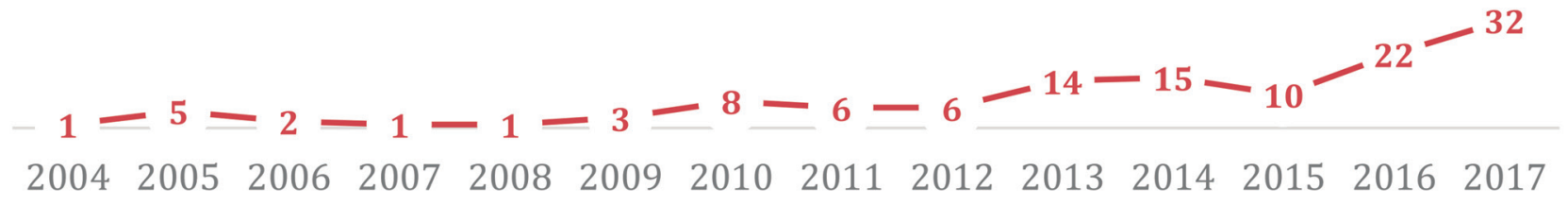

$$
\begin{aligned}
& \text { - - Total manuscritos publicados } \quad \text { - }- \text { Total manuscritos extrajeros }
\end{aligned}
$$

Figura 2. Número de manuscritos extranjeros publicados 2004-2017(incluyendo suplementos)

autores como revisores de los manuscritos. El año 2017 participaron activamente 178 revisores, que realizaron en total 300 revisiones de manuscritos.

Algunos de los principales hitos que han llevado a la revista a su posición actual son el trabajo editorial en una plataforma profesional como es el Open Journal System (OJS), la publicación bilingüe de la revista, la publicación de los artículos próximos con su Identificador Digital DOI, acortando significativamente los tiempos editoriales desde que un manuscrito es recibido hasta que es publicado, el código QR, a lo cual se ha sumado la herramienta Crossref para pesquisa de plagio.

La profesionalización del trabajo editorial es un desafío constante para mantener la Revista en su estándar actual y para continuar con su desarrollo.

\section{Conclusiones}

Chile se encuentra en altos niveles en cuanto a su producción científica, superando los demás países de la región al ajustar por número de habitantes o PBI.
Revista Chilena de Pediatría aspira a continuar siendo una opción de publicación principal para la investigación relacionada con la infancia a nivel nacional, regional y también para investigadores de otros continentes. Como revista representante de una sociedad científica como es la Sociedad Chilena de Pediatría, es fundamental mantener el equilibrio de la sustentabilidad científica y económica, además del balance entre el impacto nacional y el científico requerido para continuar ascendiendo en los ranking internacionales.

\section{Conflicto de intereses}

Los autores declaran no tener conflicto de intereses.

\section{Referencias}

1. Cienfuegos, E., \& Schonhaut, L. Distrepsias de Origen Psíquico: Publicado en Rev Chil Pediatr 1930. Rev Chil Pediatr 2007;78(5):542-8.

2. Micco S. La vida necesita ser pensada. Rev Chil Pediatr 2017;88(4):541-4. 